\title{
DESARROLLO DE UNA APLICACIÓN PARA EL CÁLCULO DE LAS PROPIEDADES DE LA MEZCLA AMONIACO-AGUA
}

\section{Development of a computer application for the calculation of the thermodynamic properties of the ammonia-water mixture}

\author{
Iván Vera-Romero ${ }^{1}$, Christopher L. Heard-Wade ${ }^{2}$ \\ ${ }^{1}$ M.Sc., Instituto Mexicano del Petróleo (San Bartolo Atepehuacan, México). ivanverar@gmail.com \\ 2Ph.D., Universidad Autónoma Metropolitana (Colonia Santa Fé, Cuajimalpa, México. cheard@correo.cua.uam.mx.
}

(Recibido mayo 17 de 2017 y Aprobado junio 3 de 2017)

\begin{abstract}
Resumen
El diseño y la optimización de sistemas energéticos en la actualidad son de gran importancia. Algunos de estos sistemas emplean la mezcla amoniaco-agua como fluido de trabajo, por lo tanto, el cálculo de las propiedades termodinámicas se vuelve indispensable para su evaluación, diseño y optimización. En el presente trabajo se ha desarrollado una aplicación en Excel ${ }^{\mathrm{TM}}$ empleando Visual Basic (VBA) a partir de una formulación basada en la energía de Gibbs de exceso, con la finalidad de simular diversos sistemas, como pueden ser de refrigeración, aire acondicionado, bombas de calor, cogeneración y ciclos de potencia, sin la necesidad de adquirir simuladores comerciales para tal propósito. Para validar dicho programa se han comparado los resultados con los datos arrojados por el software desarrollado por el National Institute of Standars and Technology (NIST) y datos experimentales reportados en literatura.
\end{abstract}

Palabras clave: mezcla amoniaco-agua; propiedades termodinámicas; energía libre de Gibbs de exceso; entalpía; entropía.

\begin{abstract}
The design and optimization of energy systems are very important today. Some of these systems use the ammonia-water mixture as working fluid, therefore, calculation of the thermodynamic properties becomes indispensable for its evaluation, design and optimization. In the present work an application has been developed in Excel ${ }^{\mathrm{TM}}$ using Visual Basic (VBA) from a formulation based on free Gibbs Energy of Excess, in order to simulate different systems such as cooling, air conditioning, heat pumps, cogeneration and power cycles, without to acquire commercial simulators for this purpose. To validate this program, the results were compared with data obtained by the National Institute of Standards and Technology (NIST) software and experimental data reported in the literature.
\end{abstract}

Keywords: Ammonia-water mixture; thermodynamic properties; Gibb free energy; enthalpy; entropy.

\section{INTRODUCCIÓN}

La mezcla amoniaco-agua (MAW) ha sido extensamente utilizada en diversos sistemas energéticos, principalmente en sistemas de refrigeración por absorción (SRA) y ciclos de potencia. A partir de la década de los setenta, el interés por estudiar más a fondo esta mezcla se intensificó debido a la denominada crisis energética que propició el incremento de los sistemas de ahorro de energía en la industria. La MAW ha tenido una implicación importante para el ahorro de energía, principalmente en el uso de bombas de calor o como SRA al aplicarse $y$, principalmente, al acoplarse a algunos equipos externos como lo han sido torres de destilación [1], el ciclo Kalina $[2,3]$, sistemas de climatización y como medios de enfriamiento de aire para las turbinas de gas [4].

Aunque se han encontrado trabajos experimentales para la evaluación de sus propiedades, reportados en la literatura desde la segunda mitad del siglo XIX [5], la estimación de las propiedades termodinámicas y termo-físicas [6] ha sido complicada, ya que esta mezcla 
presenta un comportamiento altamente polar. Hay que mencionar que, a pesar de esto, es esta última característica la que la hace apta para los SRA.

A lo largo del estudio de esta mezcla se han propuesto numerosos métodos para la estimación de sus propiedades. Algunos de ellos muestran un acercamiento con deficiencias considerables, principalmente cuando se usan en los límites de la estimación del modelo o cuando la concentración de amoniaco en la mezcla es alta $(x \rightarrow 1)$. Los análisis y diseño de los SRA, en su gran mayoría, aproximadamente en la primera mitad del siglo pasado se basaron en predicciones gráficas, sin embargo el intervalo de presiones no era tan amplio, pues se basaba en pocos datos experimentales y en deducciones teórico-empíricas para su determinación [7]. Para el año de 1957, el Institute of Gas Technology (IGT) [8] presentó un diagrama apoyado en el trabajo reportado por George Granger Brown and Associates, el cual fue utilizado hasta antes del trabajo reportado por [7], donde este último se enfocó en resolver el problema del desconocimiento de la concentración o cantidad de agua en el vapor a la salida del generador. Dado que el precisar la cantidad de agua en la fase vapor del refrigerante saliendo del generador o rectificador (desorbedor) conlleva en su gran mayoría un sobrediseño o el déficit del mismo [6]. Este último trabajo amplió la experimentación de $2.068 \mathrm{MPa}$ a $3.45 \mathrm{MPa}$ en todo el intervalo de concentración. Lo cual representó, hasta entonces y durante algún tiempo después, la base de consulta para la mayoría de ingenieros e investigadores $[5,9]$ como base de cálculos para el diseño y evaluación de SRA, debido a que cubría todo el intervalo de concentración a presiones y temperaturas adecuadas para estos sistemas.

Posteriormente, algunos trabajos se enfocaron en la estimación de propiedades para los análisis de sistemas que involucran corrientes no necesariamente para fines de refrigeración. En este caso puede tratarse de procesos en industrias extractivas como carbón o petróleo, donde algunas de sus corrientes de procesos en sus diferentes etapas contienen la solución de amoniaco-agua, por lo tanto, su análisis no necesariamente es a temperaturas cercanas a cero [10]. Por otro lado, se encuentra el ciclo Kalina, el cual se analiza a temperaturas y presiones mayores que un sistema convencional [3].

Por lo tanto, contar con modelos matemáticos que predigan las propiedades termodinámicas de esta mezcla de manera más precisa, es realmente importante para el estudio del funcionamiento de los SRA y las posibles mejoras que se puedan realizar. En las últimas décadas, los análisis de estos sistemas no solo se han orientado a conocer su desempeño mediante la primera ley de la termodinámica, sino para determinar también sus irreversibilidades, mediante la implementación del análisis por segunda ley. Este último involucra el valor de la entropía, la cual es fundamental para una mejor estimación del desempeño del SRA y su optimización $[1,3,11,50]$.

Teniendo en cuenta que la mezcla de amoniaco y agua forma una solución altamente no ideal, se ha hecho complejo el modelado de su comportamiento, principalmente cuando el análisis se extiende cerca de la región crítica o cuando la concentración del amoniaco sobrepasa el 97 \% en la mezcla. La desventaja de algunos modelos desarrollados es que trabajan dentro de un intervalo reducido, otro inconveniente es que no estiman la entropía $[12,13]$. Actualmente, y con la necesidad de efectuar balances de exergia [56], se ha recurrido a las ecuaciones cúbicas de estado, establecidas en los simuladores comerciales para el análisis de estos sistemas $[1,11]$. Sin embargo, no cuentan con las adecuaciones necesarias que exige esta mezcla. Por esta razón, muchos investigadores se han dado a la tarea de modificar o generar ecuaciones especializadas, aunque son pocos los modelos con una gama amplia de aplicación [14].

Para la estimación de las propiedades termodinámicas de la MAW se pueden catalogar más de 50 metodologías publicadas [9,13,15-33,35-37], las cuales, a su vez, se han dividido en siete grupos principales [15]: ecuación cúbica de estado, ecuación viral de estado, energía de Gibbs de exceso, ley de estados correspondientes, teoría de perturbación, método de contribución de grupos y funciones polinómicas. Como una contribución adicional, aunque poco explorada, se puede mencionar un octavo grupo: redes neuronales artificiales [34,48].

En los últimos años, la obtención de las propiedades termodinámicas de esta mezcla por medio de la programación se ha incrementado como un reflejo de la necesidad de simular sistemas energéticos $[37,47,49,51$ 54].

Debido a lo anterior, la motivación de este trabajo es contar con una herramienta confiable capaz de simular 
fenómenos que involucren la MAW, y que pueda ser programada sin necesidad de adquirir un software comercial para diferentes aplicaciones académicas [55] y de ingeniería aplicada $[38,39]$. Por tanto, el siguiente trabajo tiene como objeto la programación y validación de la metodología expuesta por [25] para su aplicación en la simulación de SRA de bajas y medianas presiones, aire acondicionado [40] y ciclos de potencia como el ciclo Kalina [41]. Se elige esta correlación debido a que tiene la ventaja de obtener las propiedades bajo condiciones críticas (Pc y Tc), así como las temperaturas de punto de burbuja (Tb) y de rocío (Td), de una manera rápida y sencilla a través de la soluciones de polinomios, sin la necesidad de estimar la fugacidad por métodos complicados.

Se hace una comparación contra los resultados arrojados por el software Reference Fluid Thermodynamic and Transport Properties versión 8.0 (REFPROP v.8.0) desarrollado por el National Institute of Standars and Technology (NIST), basado en el modelo presentado por [9], y datos experimentales reportados en literatura. Se creó una herramienta en Excel ${ }^{\mathrm{TM}}$ Add-In a través de Visual Basic (VBA Project) extensión "*xla", donde se programaron las funciones para obtener las propiedades termodinámicas.

\section{MODELO MATEMÁTICO SELECCIONADO}

Para este trabajo se empleó una metodología basada en la energía de Gibbs de exceso, la cual fue propuesta por [25], con base en el trabajo de [42] con una variación en el cálculo del coeficiente de actividad para la determinación de las temperaturas de burbuja y rocío, en donde se sustituye por el modelo de [43]. Toda esta metodología es un trabajo de actualización de la metodología propuesta por [3] en 1989, la cual combinó el trabajo de [44] con el de $[43,14]$. A continuación se describe la formulación completa empleada en la programación (ecuaciones 1-28).

Propiedades para el componente puro,

Energía libre de Gibbs para un componente puro,

$$
\begin{aligned}
& G=h_{0}-T s_{0}+\int_{T 0}^{T} C p d T+\int_{P 0}^{P} v d P- \\
& T \int_{T 0}^{T}(C p / T) d T
\end{aligned}
$$

Volumen específico y calor específico a presión constante, para ambas fases,

$$
\begin{aligned}
& v^{L}=A_{1}+A_{2} P+A_{3} T+A_{4} T^{2} \\
& c_{p}^{L}=B_{1}+B_{2} T+B_{3} T^{2} \\
& v^{g}=\frac{R T}{P}+C_{1}+\frac{C_{2}}{T^{3}}+\frac{C_{3}}{T^{11}}+\frac{C_{4} P^{2}}{T^{11}} \\
& c_{p}^{g}=D_{1}+D_{2} T+D_{3} T^{2}
\end{aligned}
$$

Fase líquida:

$$
\begin{aligned}
& G_{r}^{L}=h_{r, 0}^{L}-T_{r} S_{r, 0}^{L}+B_{1}\left(T_{r}-T_{r, 0}\right)+\left(B_{2} / 2\right)\left(T_{r}^{2}-\right. \\
& \left.T_{r, 0}^{2}\right)+\left(B_{3} / 3\right)\left(T_{r}^{3}-T_{r, 0}^{3}\right)-B_{1} T_{r} \ln \left(T_{r} / T_{r, 0}\right)- \\
& B_{2} T_{r}\left(T_{r}-T_{r, 0}\right)-\left(B_{3} / 2\right) T_{r}\left(T_{r}^{2}-T_{r, 0}^{2}\right)+ \\
& \left(A_{1}+A_{3} T_{r}+A_{4} T_{r}^{2}\right)\left(P_{r}-P_{r, 0}\right)+\left(A_{2} / 2\right)\left(P_{r}^{2}-\right. \\
& \left.P_{r, 0}^{2}\right)
\end{aligned}
$$

Fase gaseosa:

$$
\begin{aligned}
& G_{r}^{g}=h_{r, 0}^{g}-T_{r} s_{r, 0}^{g}+D_{1}\left(T_{r}-T_{r, 0}\right)+\left(D_{2} / 2\right)\left(T_{r}^{2}-\right. \\
& \left.T_{r, 0}^{2}\right)+\left(D_{3} / 3\right)\left(T_{r}^{3}-T_{r, 0}^{3}\right)-D_{1} T_{r} \ln \left(T_{r} / T_{r, 0}\right)- \\
& D_{2} T_{r}\left(T_{r}-T_{r, 0}\right)-\left(D_{3} / 2\right) T_{r}\left(T_{r}^{2}-T_{r, 0}^{2}\right)+ \\
& T_{r} \ln \left(P_{r} / P_{r, 0}\right)+C_{1}\left(P_{r}-P_{r, 0}\right)+C_{2}\left(P_{r} / T_{r}^{3}-\right. \\
& \left.4 P_{r, 0} / T_{r, 0}^{3}+3 P_{r, 0} T_{r} / T_{r, 0}^{4}\right)+C_{3}\left(P_{r} / T_{r}^{11}-\right. \\
& \left.12 P_{r, 0} / T_{r, 0}^{11}+11 P_{r, 0} T_{r} / T_{r, 0}^{12}\right)+\left(C_{4} / 3\right)\left(P_{r}^{3} / T_{r}^{11}-\right. \\
& \left.12 P_{r, 0}^{3} / T_{r, 0}^{11}-11 P_{r, 0}^{3} T_{r} / T_{r, 0}^{12}\right)
\end{aligned}
$$

donde,

$$
\begin{aligned}
& T_{r}=T / T_{B} \\
& P_{r}=P / P_{B}
\end{aligned}
$$

$$
G_{r}=G / R T_{B}
$$

$$
h_{r}=h / R T_{B}
$$

$s_{r}=s / R$ 
$v_{r}=v P_{B} / T_{B}$

$R=8.314 \mathrm{~kJ} / \mathrm{kmol} \mathrm{K}$

$$
\begin{aligned}
& s^{E}=-R\left[\frac{\partial G_{r}^{E}}{\partial T_{r}}\right]_{P_{r, x}} \\
& v^{E}=\frac{R T_{B}}{P_{B}}\left[\frac{\partial G_{r}^{E}}{\partial P_{r}}\right]_{T_{r, x}}
\end{aligned}
$$

$P_{B}=10 \mathrm{bar}$

Propiedades termodinámicas para un componente puro,

$h=-R T_{B} T_{r}^{2}\left[\frac{\partial}{\partial T_{r}}\left(G_{r} / T_{r}\right)\right]_{P_{r}}$

$s=-R\left[\frac{\partial G_{r}}{\partial T_{r}}\right]_{P_{r}}$

$v=\frac{R T_{B}}{P_{B}}\left[\frac{\partial G_{r}}{\partial P_{r}}\right]_{T_{r}}$

Entalpía, entropía y volumen específico para la mezcla líquida,

$h_{m}^{L}=x_{f} h_{a}^{L}+\left(1-x_{f}\right) h_{w}^{L}+h^{E}$

$s_{m}^{L}=x_{f} s_{a}^{L}+\left(1-x_{f}\right) s_{w}^{L}+s^{E}+s^{m i x}$

$v_{m}^{L}=x_{f} v_{a}^{L}+\left(1-x_{f}\right) v_{w}^{L}+v^{E}$

Entalpía, entropía y volumen específico para la mezcla gaseosa,

$h_{m}^{g}=x_{g} h_{a}^{g}+\left(1-x_{g}\right) h_{w}^{g}$ amoniaco-agua,

$s_{m}^{g}=x_{g} s_{a}^{g}+\left(1-x_{g}\right) s_{w}^{g}+s^{m i x}$

$G_{r}^{E}=(1-x) x\left[F_{1}+F_{2}(2 x-1)+F_{3}(2 x-1)^{2}\right]$

donde x es la fracción masa del amoniaco,

$v_{m}^{g}=x_{g} v_{a}^{g}+\left(1-x_{g}\right) v_{w}^{g}$

donde,

$F_{1}=E_{1}+E_{2} P_{r}+\left(E_{3}+E_{4} P_{r}\right) T_{r}+E_{5} / T_{r}+E_{6} / T_{r}^{2}(12)$

$F_{2}=E_{7}+E_{8} P_{r}+\left(E_{9}+E_{10} P_{r}\right) T_{r}+E_{11} / T_{r}+$

$E_{12} / T_{r}^{2}$

$F_{3}=E_{13}+E_{14} P_{r}+E_{15} / T_{r}+E_{16} / T_{r}^{2}$

$s^{m i x}=-R[x \ln (x)+(1-x) \ln (1-x)]$

Para las propiedades de burbuja, rocío y críticas, se emplean las siguientes ecuaciones (25-28).

$T_{b}=T_{c}-\sum_{i=1}^{7}\left(C_{i}+\sum_{j=1}^{10} C_{i j} x^{j}\right)\left[\ln \left(P_{c} / P\right)\right]^{i}$

$h^{E}=-R T_{B} T_{r}^{2}\left[\frac{\partial}{\partial T_{r}}\left(G_{r}^{E} / T_{r}\right)\right]_{P_{r, x}}$ 


$$
\begin{gathered}
T_{d}=T_{c}- \\
\sum_{i=1}^{6}\left(a_{i}+\sum_{j=1}^{4} A_{i j}[\ln (1.0001-x)]^{j}\right)\left[\ln \left(P_{c} / P\right)\right]^{i}
\end{gathered}
$$

$T_{c}=T_{c w}-\sum_{i=1}^{4} a_{c r i t, i} x^{i}$

$P_{c}=P_{c w} \exp \left(\sum_{i=1}^{8} b_{c r i t, i} x^{i}\right)$

Nota: $\mathrm{P}$ en psia y $\mathrm{T}$ en ${ }^{\circ} \mathrm{F}$, para las ecuaciones 27 y 28. Mientras que $x$ es la fracción mol de amoniaco en la mezcla. Los coeficientes para las condiciones de la mezcla se pueden apreciar en las Tablas 1 y 2.

Tabla 1. Coeficientes y estados de referencia para ecuaciones (2-7)

\begin{tabular}{lll}
\hline Coeficiente & Amoniaco & Agua \\
\hline $\mathrm{A}_{1}$ & 0.03971423 & 0.02748796 \\
$\mathrm{~A}_{2}$ & $-1.790557 \mathrm{E}-05$ & $-1.016665 \mathrm{E}-05$ \\
$\mathrm{~A}_{3}$ & -0.01308905 & -0.004452025 \\
$\mathrm{~A}_{4}$ & 0.003752836 & 0.000838925 \\
$\mathrm{~B}_{1}$ & 16.34519 & 12.14557 \\
$\mathrm{~B}_{2}$ & -6.508119 & -1.898065 \\
$\mathrm{~B}_{3}$ & 1.448937 & 0.2911966 \\
$\mathrm{C}_{1}$ & -0.01049377 & 0.02136131 \\
$\mathrm{C}_{2}$ & -8.288224 & -31.69291 \\
$\mathrm{C}_{3}$ & -664.7257 & -46346.11 \\
$\mathrm{C}_{4}$ & -3045.352 & 0.0 \\
$\mathrm{D}_{1}$ & 3.673647 & 4.01917 \\
$\mathrm{D}_{2}$ & 0.09989629 & -0.0517555 \\
$\mathrm{D}_{3}$ & 0.03617622 & 0.01951939 \\
$h_{r, 0}^{L}$ & 4.878573 & 21.821141 \\
$h_{r, 0}^{g}$ & 26.468873 & 60.965058 \\
$s_{r, 0}^{L}$ & 1.644773 & 5.733498 \\
$s_{r, 0}^{g}$ & 8.339026 & 13.45343 \\
$T_{r, 0}$ & 3.2252 & 5.0705 \\
$P_{r, 0}$ & 2.000 & 3.000 \\
\hline
\end{tabular}

Tabla 2. Coeficientes para las propiedades de exceso, ecuaciones (12-14)

\begin{tabular}{lrlr}
\hline E1 & -41.733398 & E9 & 0.387983 \\
E2 & 0.02414 & E10 & -0.004772 \\
E3 & 6.702285 & E11 & -4.648107 \\
E4 & -0.011475 & E12 & 0.836376 \\
E5 & 63.608967 & E13 & -3.553627 \\
E6 & -62.490768 & E14 & 0.000904 \\
E7 & 1.761064 & E15 & 24.361723 \\
E8 & 0.008626 & E16 & -20.736547 \\
\hline
\end{tabular}

Las ecuaciones para las propiedades termodinámicas fueron derivadas, y programadas con ayuda de Excel $^{\mathrm{TM}}$ y Visual Basic Application (VBA), por medio de la creación de subrutinas en un proyecto "xla", el cual es un complemento para Excel ${ }^{\mathrm{TM}}$. Donde se generaron funciones capaces de resolver las distintas fases y concentraciones que puede presentar la MAW: líquido comprimido, vapor sobrecalentado, mezcla líquidovapor, y líquido y vapor saturados (Figura 1). Las funciones desarrolladas en la programación se clasifican en directas e indirectas, como se puede apreciar en la Tabla 3. Las directas obedecen a una resolución directa de las ecuaciones, en cambio las indirectas obedecen 
a la implementación de un método iterativo para su resolución, en este caso se empleó el método por bisección (ecuaciones 29-33).

Algoritmo para el método de bisección, con valores iniciales inferior y superior $x_{i} \leq x_{r} \leq x_{f}[45]$.

Verificación,

$f\left(x_{i}\right) f\left(x_{f}\right)<0$

Aproximación de la raíz $\mathrm{x}_{\mathrm{r}}$

$x_{r}=\frac{x_{i}+x_{f}}{2}$

$\mathrm{Si}$

$f\left(x_{i}\right) f\left(x_{r}\right)<0, x_{f}=x_{r}$, por lo tanto realizar verificación.

$f\left(x_{i}\right) f\left(x_{r}\right)>0, x_{i}=x_{r}$, por lo tanto realizar verificación.

$f\left(x_{i}\right) f\left(x_{r}\right)=0$, la raíz es igual a $x_{r}$, termina el cálculo.

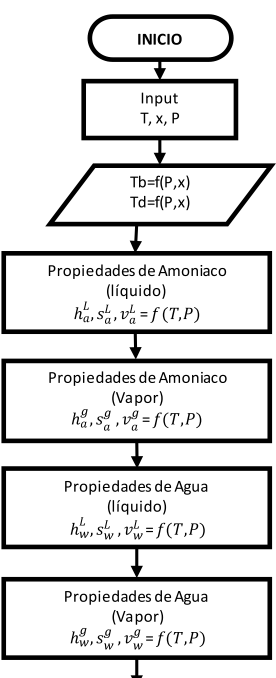

1

Propiedades de la Mezcla

(Líquido y Vapor)

$h_{m}^{L}, s_{m}^{L}, v_{m}^{L}=f(T, P, x)$
$h_{m}^{g}, s_{m}^{g}, v_{m}^{g}=f(T, P, x)$

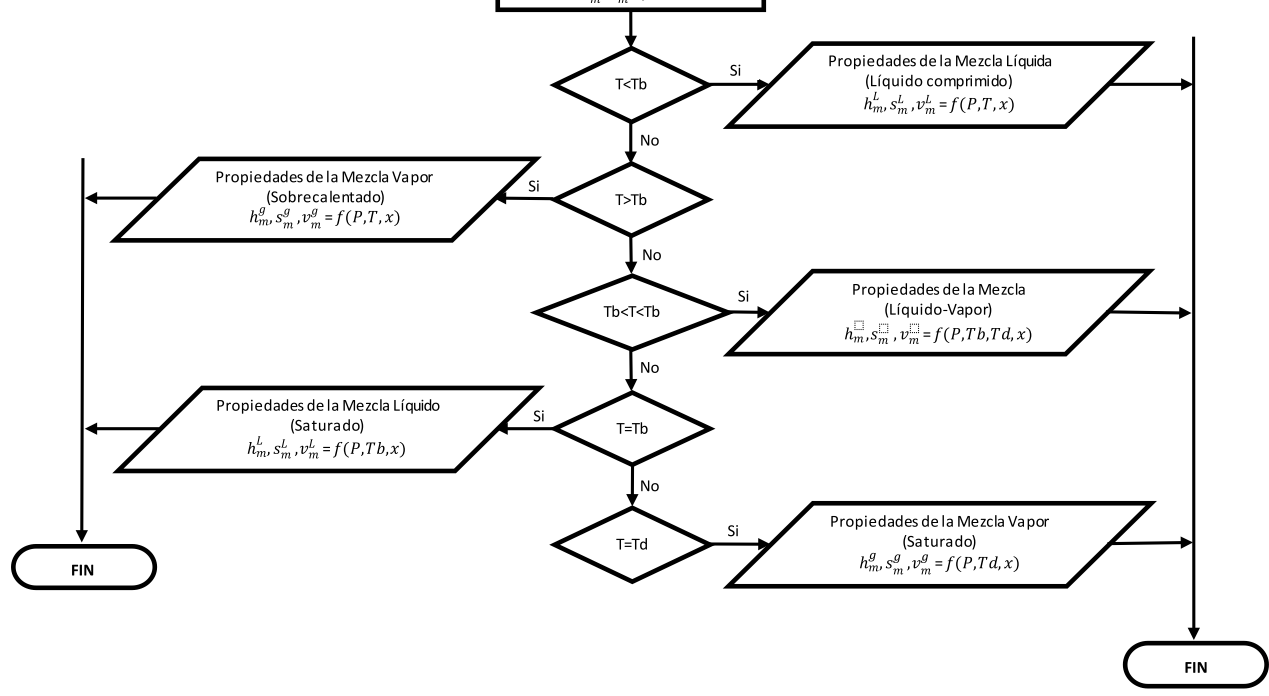

Figura 1. Diagrama de flujo para la obtención de las propiedades entalpía, entropía y volumen específico de la MAW. 
Tabla 3. Funciones programadas para el cálculo de las propiedades

\begin{tabular}{|c|c|c|c|}
\hline \multicolumn{2}{|l|}{ Función directa } & \multicolumn{2}{|r|}{ Función indirecta } \\
\hline Variable Dependiente & $\begin{array}{c}\text { Variable } \\
\text { Independiente }\end{array}$ & $\begin{array}{c}\text { Variable } \\
\text { Dependiente }\end{array}$ & Variable Independiente \\
\hline Temperatura líquido Comprimido $(\mathrm{T})$ & $\begin{array}{l}\text { Presión }(\mathrm{P}) \\
\text { Concentración } \\
\text { amoniaco }(\mathrm{x})\end{array}$ & $\begin{array}{l}\text { Presión Líquido } \\
\text { Saturado (P) }\end{array}$ & $\begin{array}{l}\text { Concentración de amoniaco }(x) \\
\text { Temperatura (Tb) }\end{array}$ \\
\hline Temperatura líquido Saturado (Tb) & $\begin{array}{l}\text { Presión de saturación } \\
(\mathrm{P}) \\
\text { Concentración } \\
\text { amoniaco }(\mathrm{x})\end{array}$ & $\begin{array}{l}\text { Concentración de } \\
\text { amoniaco (Fase } \\
\text { vapor) }\end{array}$ & $\begin{array}{l}\text { Concentración de amoniaco }(x) \\
\text { Presión }(P)\end{array}$ \\
\hline Temperatura Vapor Sobrecalentado (T) & $\begin{array}{l}\text { Presión }(\mathrm{P}) \\
\text { Concentración } \\
\text { amoniaco }(\mathrm{x})\end{array}$ & $\begin{array}{l}\text { Presión Vapor } \\
\text { Saturado (P) }\end{array}$ & $\begin{array}{l}\text { Concentración amoniaco (fase vapor) } \\
\text { Temperatura (Td) }\end{array}$ \\
\hline Temperatura Vapor Saturado (Td) & $\begin{array}{l}\text { Presión de saturación } \\
(\mathrm{P}) \\
\text { Concentración } \\
\text { amoniaco }(\mathrm{x})\end{array}$ & $\begin{array}{l}\text { Concentración de } \\
\text { amoniaco (x) }\end{array}$ & $\begin{array}{l}\text { Presión }(\mathrm{P}) \\
\text { Temperatura }(\mathrm{T})\end{array}$ \\
\hline $\begin{array}{l}\text { Temperatura de la Mezcla Líquido- } \\
\text { Vapor }(T)\end{array}$ & $\begin{array}{l}\text { Presión }(\mathrm{P}) \\
\text { Concentración de } \\
\text { amoniaco }(\mathrm{x}) \\
\end{array}$ & $\begin{array}{l}\text { Concentración de } \\
\text { amoniaco (Fase } \\
\text { vapor) }\end{array}$ & $\begin{array}{l}\text { Presión }(\mathrm{P}) \\
\text { Temperatura }(\mathrm{T})\end{array}$ \\
\hline $\begin{array}{l}\text { Entalpía, entropía y volumen específico } \\
\text { Líquido Comprimido }\left(h_{m}^{L}, s_{m}^{L}, v_{m}^{L}\right)\end{array}$ & $\begin{array}{l}\text { Presión }(\mathrm{P}) \\
\text { Temperatura }(\mathrm{T}) \\
\text { Concentración }(\mathrm{x}) \\
\end{array}$ & $\begin{array}{l}\text { Fracción masa de } \\
\text { la mezcla }(z)\end{array}$ & $\begin{array}{l}\text { Fracción de amoniaco (Fase vapor) } \\
\text { Fracción amoniaco (Fase Líquida) }\end{array}$ \\
\hline $\begin{array}{l}\text { Entalpía, entropía y volumen específico } \\
\text { Líquido Saturado }\left(h_{m}^{L}, s_{m}^{L}, v_{m}^{L}\right)\end{array}$ & $\begin{array}{l}\text { Presión }(\mathrm{P}) \\
\text { Temperatura }(\mathrm{Tb}) \\
\text { Concentración }(\mathrm{x})\end{array}$ & $\begin{array}{l}\text { Fracción masa } \\
\text { (Fase líquida) }\end{array}$ & $\begin{array}{l}\text { Fracción masa amoniaco (Fase vapor) } \\
\text { Temperatura }(T) \\
\text { Presión }(\mathrm{P}) \\
\text { Fracción masa de la mezcla }(\mathrm{z})\end{array}$ \\
\hline $\begin{array}{l}\text { Entalpía, entropía y volumen específico } \\
\text { Vapor Sobrecalentado }\left(h_{m}^{g}, s_{m}^{g}, v_{m}^{g}\right)\end{array}$ & $\begin{array}{l}\text { Presión }(\mathrm{P}) \\
\text { Temperatura }(\mathrm{T}) \\
\text { Concentración }(\mathrm{x})\end{array}$ & $\begin{array}{l}\text { Fracción masa } \\
\text { (Fase vapor) }\end{array}$ & $\begin{array}{l}\text { Fracción masa amoniaco (Fase líquido) } \\
\text { Temperatura }(T) \\
\text { Presión }(T) \\
\text { Fracción masa de la mezcla (z) }\end{array}$ \\
\hline $\begin{array}{l}\text { Entalpía, entropía y volumen específico } \\
\text { Vapor Saturado }\left(h_{m}^{g}, s_{m}^{g}, v_{m}^{g}\right)\end{array}$ & $\begin{array}{l}\text { Presión }(P) \\
\text { Temperatura }(T d) \\
\text { Concentración }(x)\end{array}$ & Calidad de vapor & $\begin{array}{l}\text { Fracción masa de la mezcla }(\mathrm{z}) \\
\text { Concentración amoniaco Fase líquida (x) } \\
\text { Concentración amoniaco (Fase vapor) }\end{array}$ \\
\hline $\begin{array}{l}\text { Entalpía, entropía y volumen específico } \\
\text { de la mezcla Líquido-Vapor }\left(h_{m}, s_{m}, v_{m}\right)\end{array}$ & $\begin{array}{l}\text { Presión }(\mathrm{P}) \\
\text { Temperatura }(\mathrm{T}) \\
\text { Concentración }(\mathrm{x}) \\
\end{array}$ & $\begin{array}{l}\text { Entalpia de la } \\
\text { mezcla }(\mathrm{Hm})\end{array}$ & $\begin{array}{l}\text { Entalpía de la mezcla (Fase líquido) } \\
\text { Entalpía de la mezcla (Fase vapor) } \\
\text { Fracción masa (Fase líquida) }\end{array}$ \\
\hline & & $\begin{array}{l}\text { Concentración } \\
\operatorname{amoniaco}(x)\end{array}$ & $\begin{array}{l}\text { Concentración de la mezcla (z) } \\
\text { Temperatura (T) } \\
\text { Entalpia de la mezcla (Hm) }\end{array}$ \\
\hline & & $\begin{array}{l}\text { Concentración } \\
\text { amoniaco (Fase } \\
\text { vapor) }\end{array}$ & $\begin{array}{l}\text { Concentración de la mezcla (z) } \\
\text { Temperatura }(T) \\
\text { Entalpia de la mezcla }(\mathrm{Hm})\end{array}$ \\
\hline
\end{tabular}

\section{RESULTADOS Y DISCUSIÓN}

La Figura 2 muestra la comparación entre modelos y datos experimentales [7, 20]. Como se observa, las diferencias son relativamente pequeñas hasta los $10 \mathrm{MPa}$ con diferencias máximas de $13 \mathrm{~K}$. Posteriormente, las diferencias se incrementan cuando las condiciones se acercan a $15 \mathrm{MPa}$ a los $14 \mathrm{~K}$ entre modelos, mientras que las diferencias en relación con los datos experimentales llegan a ser de $26 \mathrm{~K}$. 


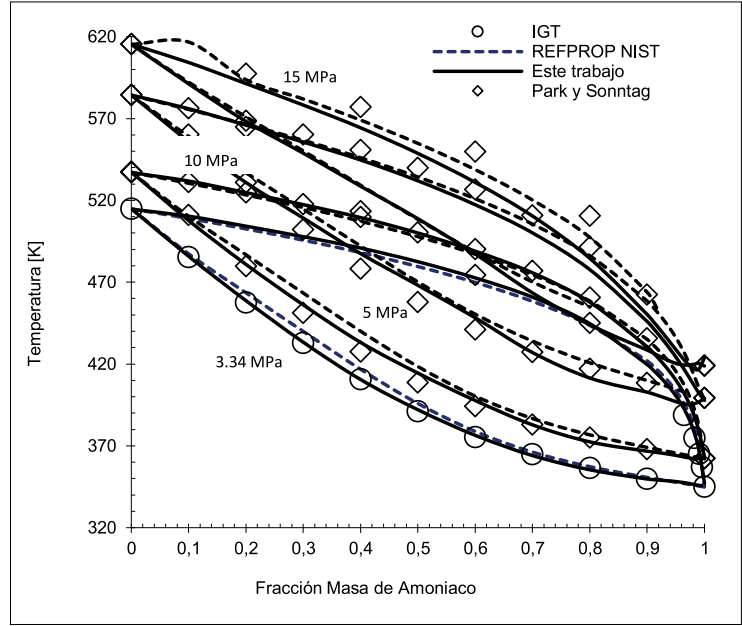

Figura 2. Temperaturas de burbuja y rocío a diferentes concentraciones y presiones.

En cuanto a la presión de saturación de la mezcla, la reproducibilidad es aceptable a $333.15 \mathrm{~K}$, la diferencia máxima entre los modelos llega a ser menor de 0.06 MPa. En la diferencia con los datos experimentales $[7,46]$, ninguno de los modelos presentó variaciones mayores a 0.3 MPa. Para el caso de $405.95 \mathrm{~K}$, las diferencias se incrementan al aumentar la concentración de amoniaco en la solución, principalmente en la zona de presión de burbuja. La diferencia entre modelos, a pesar de una notable desviación entre sus curvas, no llega a ser mayor de 1.6 MPa en la presión de líquido saturado. A su vez, para cuando la temperatura es cercana a los $423.15 \mathrm{~K}$, las diferencias se hacen más notables, manifestando que más allá de este valor los resultados pueden afectar los cálculos de evaluación de los SRA (Figura 3). Los valores de la entalpía, como se puede apreciar en las Figuras (4)-(6), poseen un comportamiento aceptable con los datos experimentales [20]. En cuanto a la entropía, se observa que hay más variación en la zona de líquido saturado que en la zona de vapor saturado. La comparación se hace, al igual que en el caso de las entalpías, entre una base de datos experimental [20], mostrando una tendencia aceptable en las tres presiones estudiadas (5 MPa, $10 \mathrm{MPa}$ y $11 \mathrm{MPa}$ ) (Figuras 7-9). Sin embargo, las diferencias se hacen más notables cuando se trabaja en las dos últimas presiones. Se hizo el análisis de volumen específico de la mezcla, tanto para líquido como para vapor saturado. En ambos casos, los modelos presentaron ajustes aceptables entre sí y con datos experimentales [46] (Figuras 10 y 11).

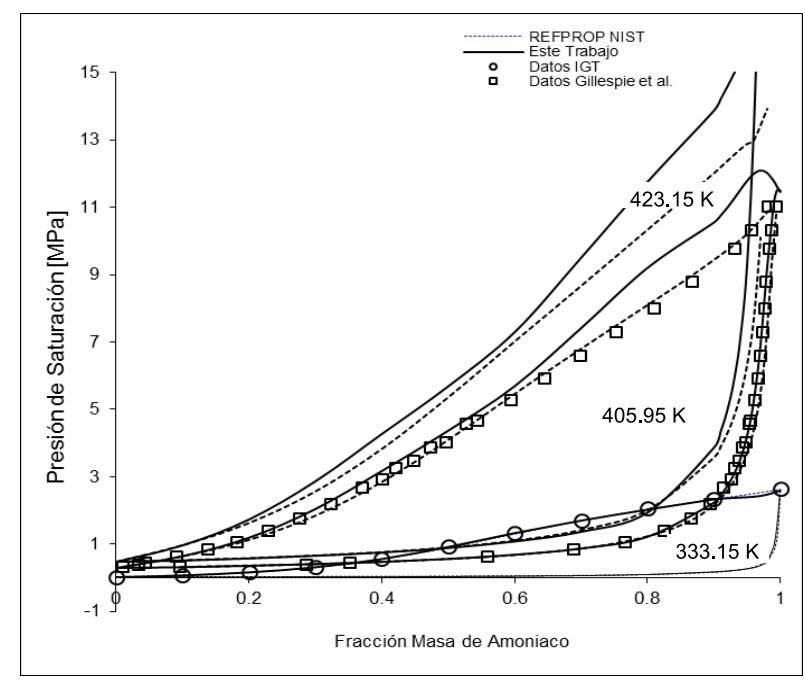

Figura 3. Presiones de saturación a tres diferentes temperaturas $(\mathrm{K})$.

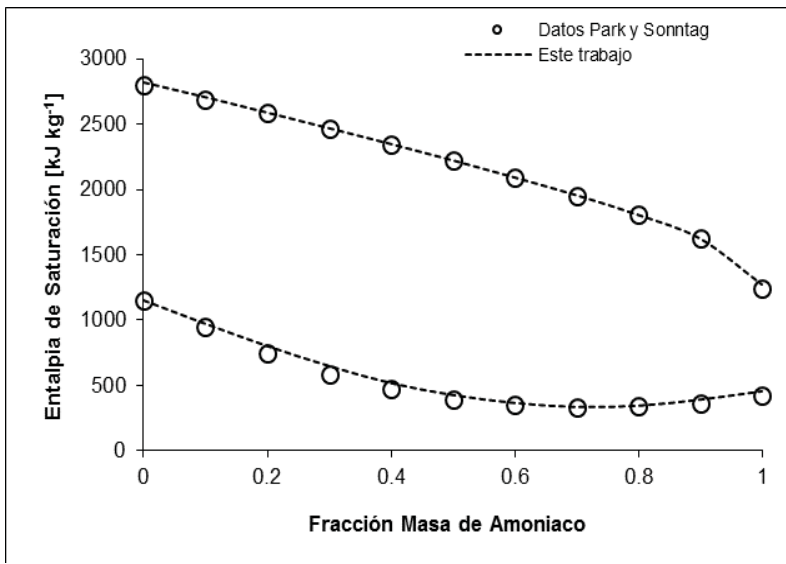

Figura 4. Entalpías de saturación a 5 MPa.

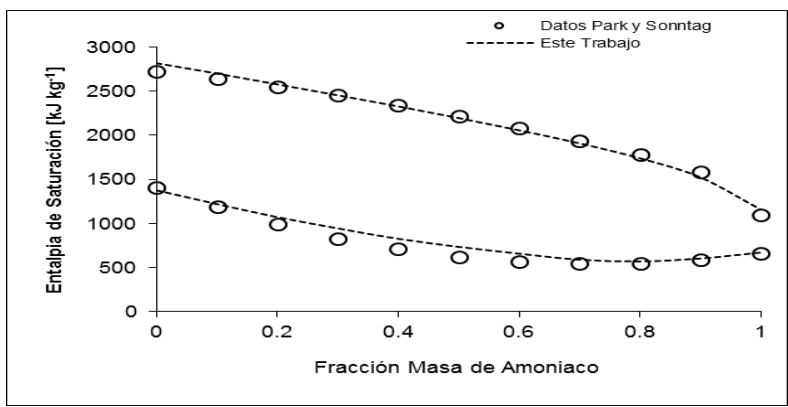

Figura 5. Entalpías de saturación a $10 \mathrm{MPa}$. 


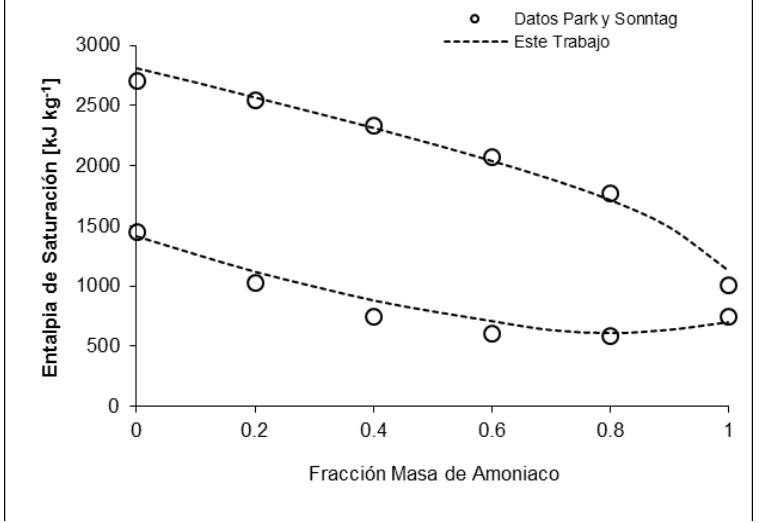

Figura 6. Entalpías de saturación a $11 \mathrm{MPa}$.

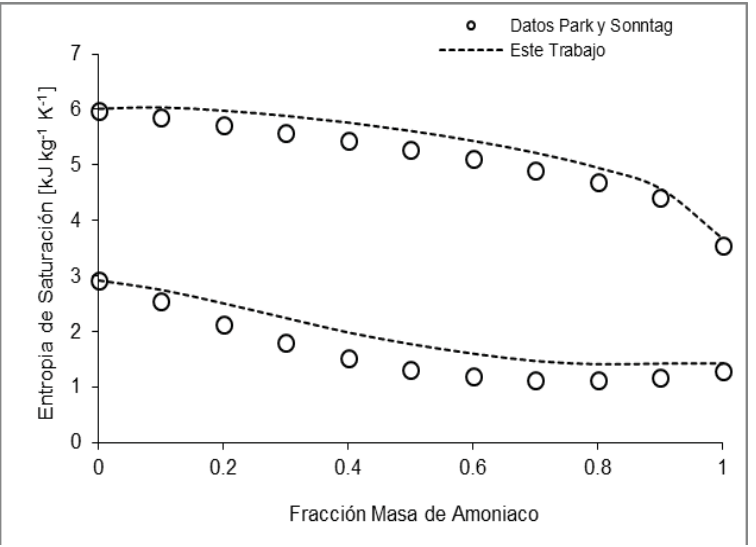

Figura 7. Entropías de saturación a $5 \mathrm{MPa}$.

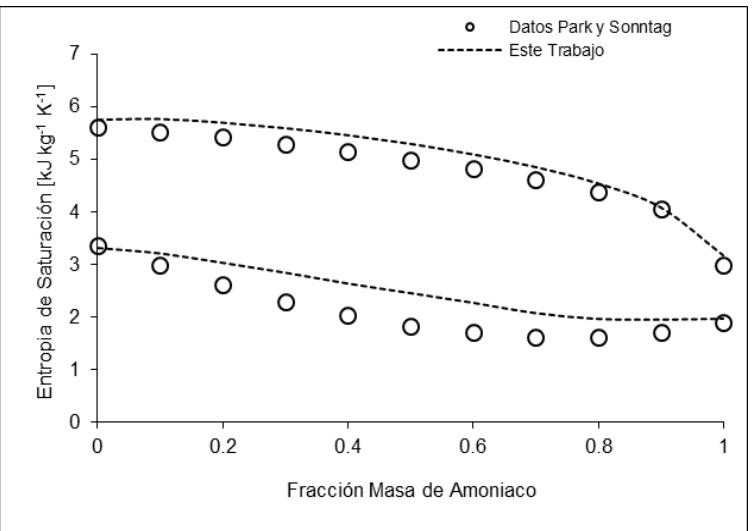

Figura 8. Entropías de saturación a $10 \mathrm{MPa}$.

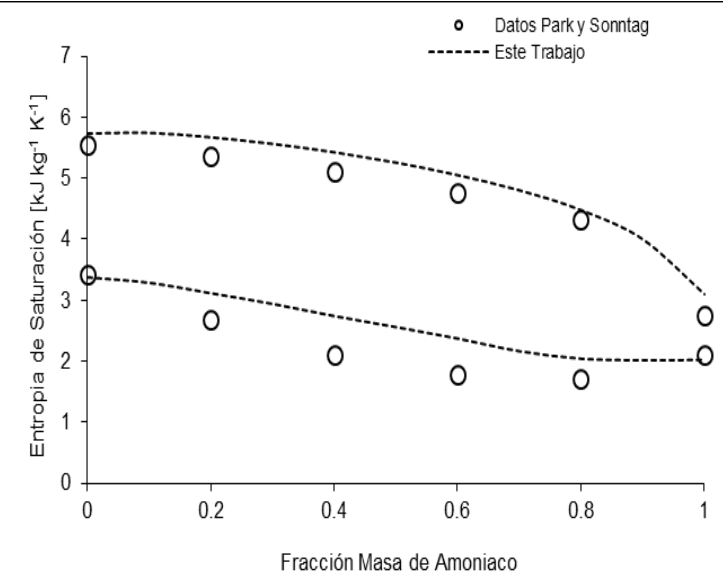

Figura 9. Entropía de saturación a $11 \mathrm{MPa}$.

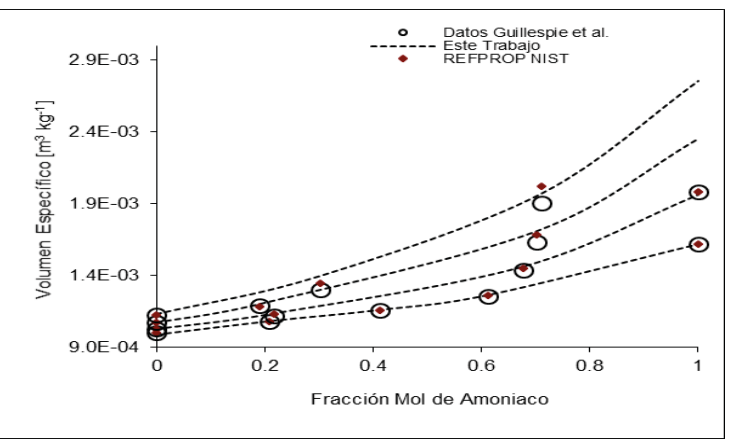

Figura 10. Volumen específico del líquido.

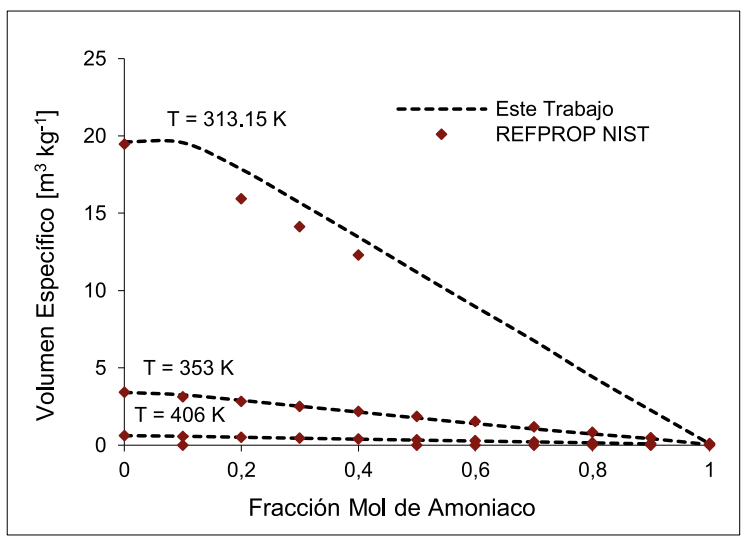

Figura 11. Volumen específico de vapor. 


\subsection{Resultados generales}

Como se ha podido apreciar, el modelo empleado en la programación presenta aceptables ajustes para las tres propiedades termodinámicas analizadas: entalpía, entropía y volumen específico. Para la entalpía, el modelo es aceptable hasta una presión de $15 \mathrm{MPa}$, lo cual significa que puede ser empleado para simulaciones por primera ley de la termodinámica hasta estas condiciones. Para la entropía, el ajuste es aceptable hasta una presión de $11 \mathrm{MPa}$, lo que significa que los análisis por segunda ley están limitados un DP de 4 MPa por debajo de los análisis por primera Ley. En cuanto al volumen específico, se puede emplear esta correlación hasta una temperatura de $450 \mathrm{~K}$. En cuanto al uso de la correlación de manera directa entre la condiciones de temperatura de burbuja y rocío contra presiones de saturación, el modelo presenta un ajuste adecuado y aceptable hasta una temperatura de $423.15 \mathrm{~K}$.

Por otro lado, las diferencias entre los modelos y los datos experimentales, y las diferencias entre los resultados de los modelos, muestran un mejor ajuste cuando las presiones no son mayores a $11 \mathrm{MPa}$ (ANEXO). Las variaciones entre las diferencias no son lineales ni tampoco muestran una tendencia predecible.

\section{CONCLUSIONES}

Se desarrolló una aplicación en Excel ${ }^{\mathrm{TM}}$ empleando VBA (*.xla) para el cálculo de las propiedades termodinámicas de la mezcla amoniaco-agua con una metodología basada en la energía de Gibbs de exceso. La comparación de las propiedades termodinámicas obtenidas contra datos experimentales y el software REFPROP (NIST) presentaron un ajuste aceptable. La aplicación es un desarrollo atractivo para el análisis de sistemas energéticos a bajas y medianas presiones, debido a su practicidad en la obtención de datos y su fácil uso, tanto para análisis por primera como por segunda ley de la termodinámica.

\section{ANEXO}

En las siguientes figuras (12-36) se observan las diferencias de las temperaturas y presiones entre los dos software empleados y datos experimentales, variando la concentración de amoniaco en la mezcla de 0 a 1.

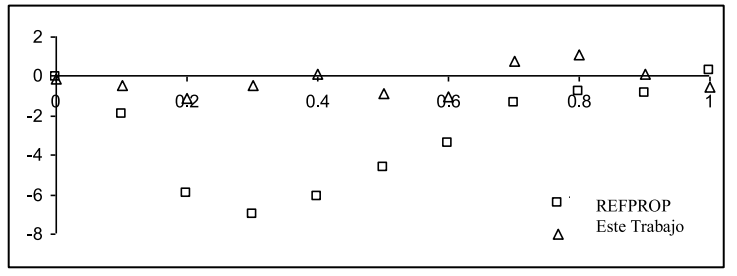

Figura 12. Diferencias para la temperatura de burbuja comparadas contra datos experimentales [7] a 3.447 $\mathrm{MPa}$.

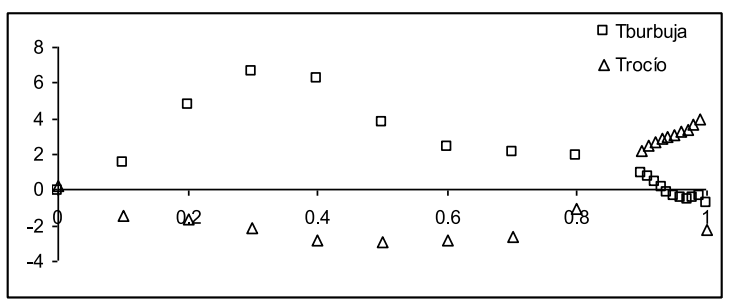

Figura 13. Diferencias comparadas entre modelos a 3.447MPa.

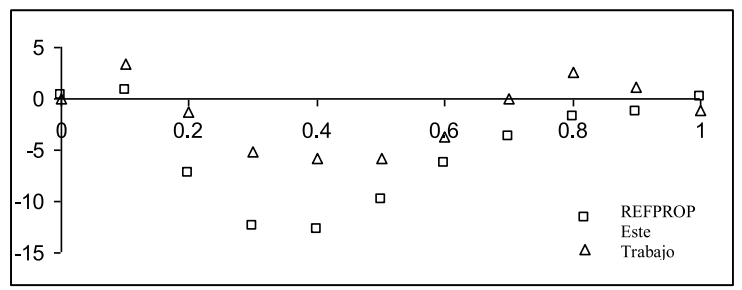

Figura 14. Diferencias para la temperatura de burbuja comparadas contra datos experimentales [7] a 5MPa.

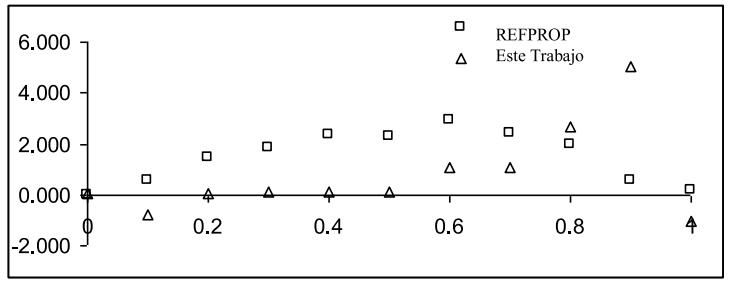

Figura 15. Diferencias para la temperatura de rocío comparadas contra datos experimentales [20] a 5MPa.

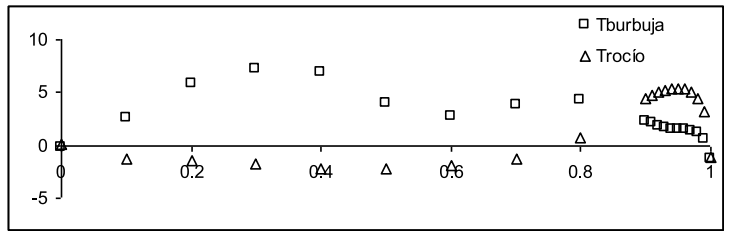

Figura 16. Diferencias comparadas entre modelos a 5MPa. 


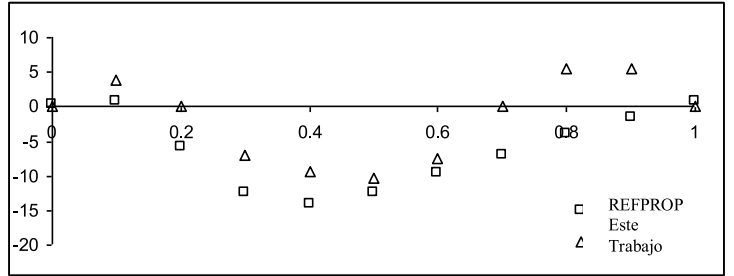

Figura 17. Diferencia para la temperatura de burbuja comparada contra datos experimentales [20] a 10MPa.

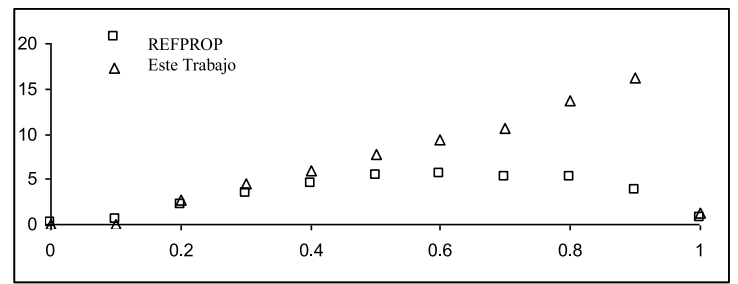

Figura 18. Diferencia para la temperatura de rocío comparada contra datos experimentales [20] a 10MPa.

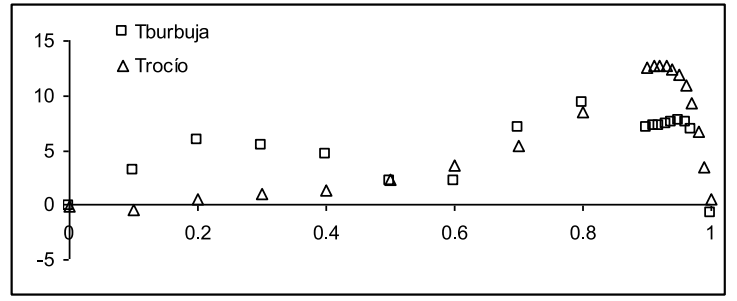

Figura 19. Diferencias comparadas entre modelos a 10MPa.

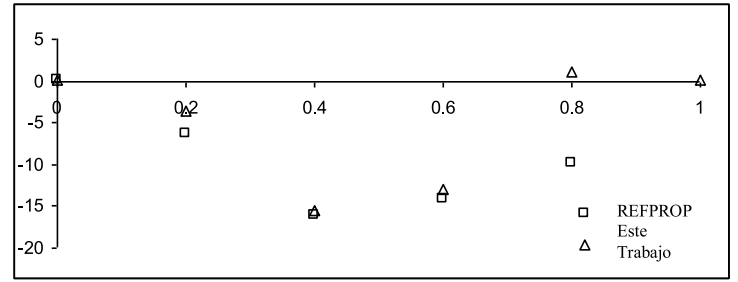

Figura 20. Diferencia para la temperatura de burbuja comparada contra datos experimentales [20] a $15 \mathrm{MPa}$.

$\begin{array}{r}30 \\ 20 \\ 10 \\ \hline\end{array}$

Figura 21. Diferencia para la temperatura de rocío comparada contra datos experimentales [20] a $15 \mathrm{MPa}$.

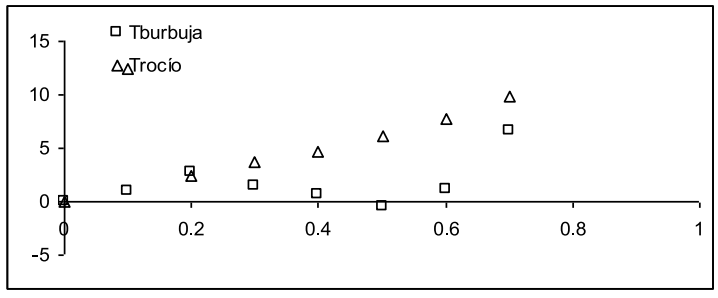

Figura 22. Diferencias comparadas entre modelos a $15 \mathrm{MPa}$.

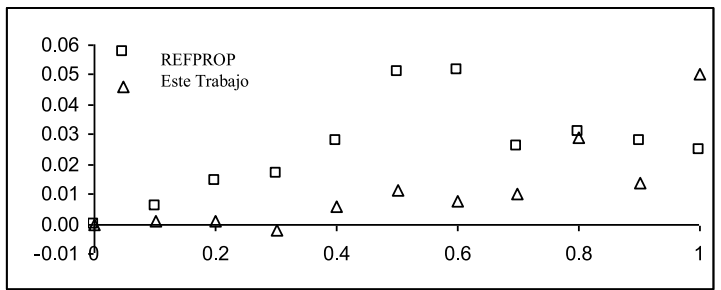

Figura 23. Diferencia para la presión de burbuja comparada contra datos experimentales [7] a 333.15MPa.

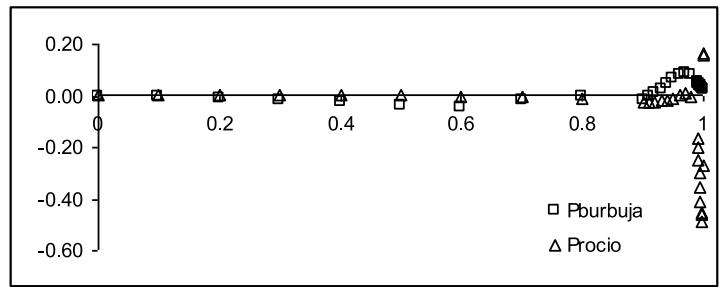

Figura 24. Diferencia de presiones comparada entre modelos a $333.15 \mathrm{~K}$.

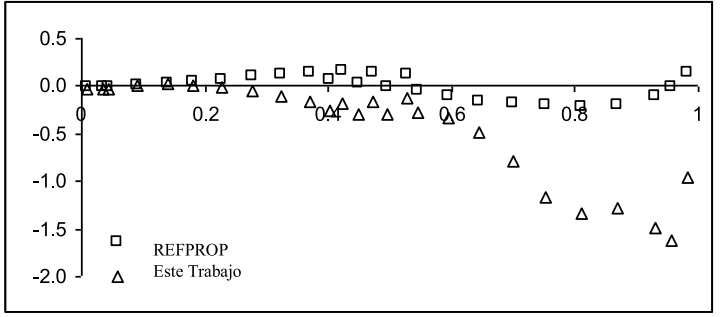

Figura 25. Diferencia para la presión de burbuja comparada contra datos experimentales [46] a 405.95K.

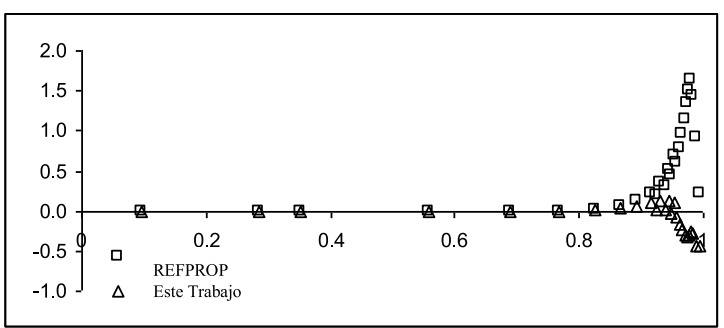

Figura 26. Diferencia para la presión de rocío comparada contra datos experimentales [46] a 405.95K. 
Desarrollo de una aplicación para el cálculo de las propiedades de la mezcla amoniaco-agua

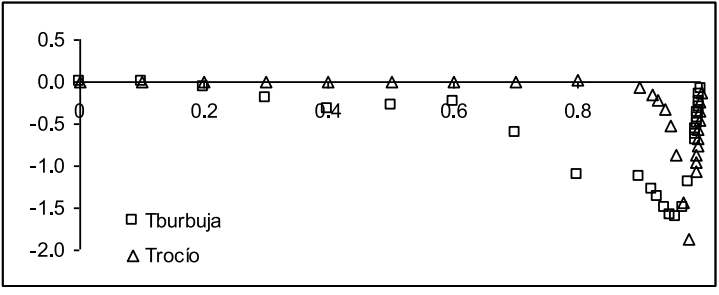

Figura 27. Diferencia de presiones comparada entre modelos a $405.95 \mathrm{~K}$.

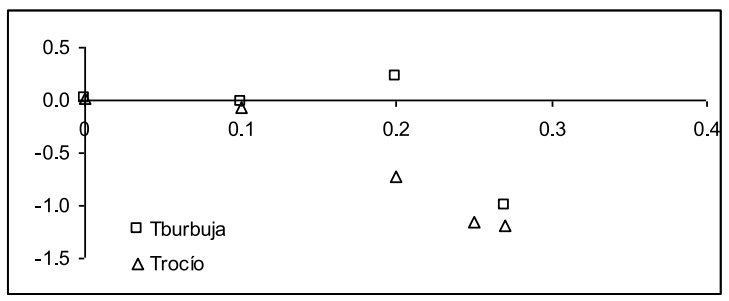

Figura 28. Diferencia de presiones comparada entre modelos a $600.00 \mathrm{~K}$.

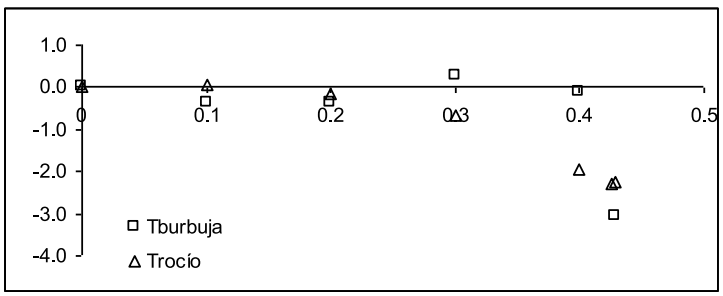

Figura 29. Diferencia de presiones comparada entre modelos a $573.15 \mathrm{~K}$.

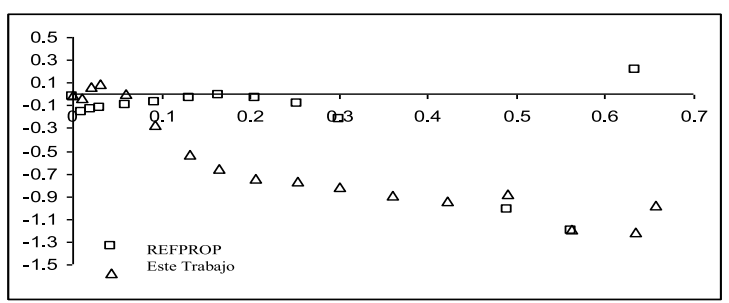

Figura 30. Diferencia para la presión de burbuja comparada contra datos experimentales [46] a 519.26K.

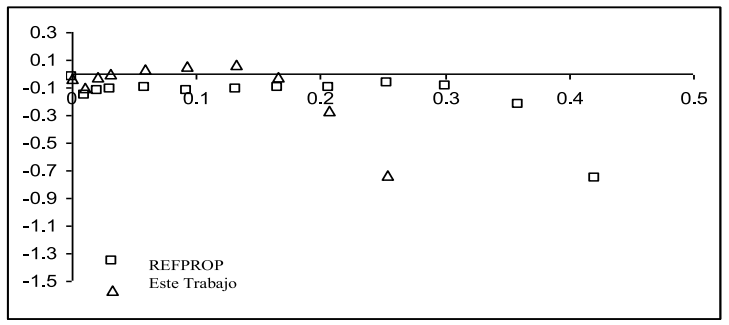

Figura 31. Diferencia para la presión de rocío comparada contra datos experimentales [46] a 519.26K.

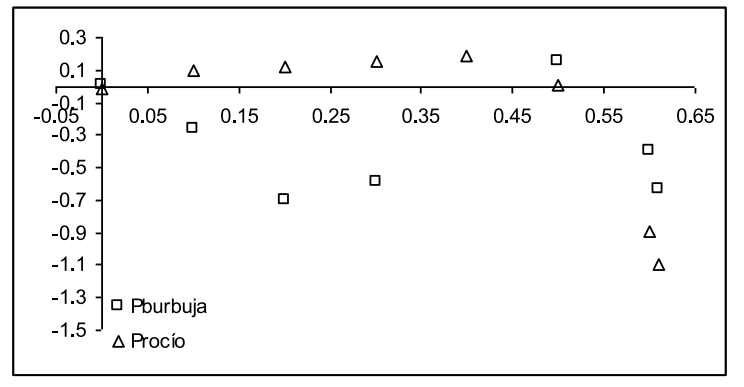

Figura 32. Diferencia de presiones comparada entre modelos a $519.26 \mathrm{~K}$.

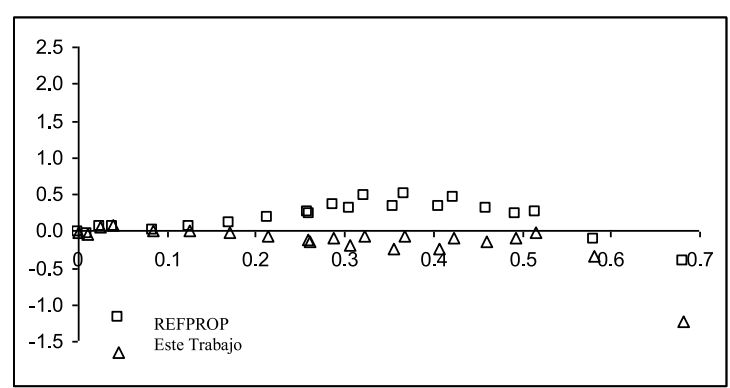

Figura 33. Diferencia para la presión de burbuja comparada contra datos experimentales [46] a $449.8 \mathrm{~K}$.

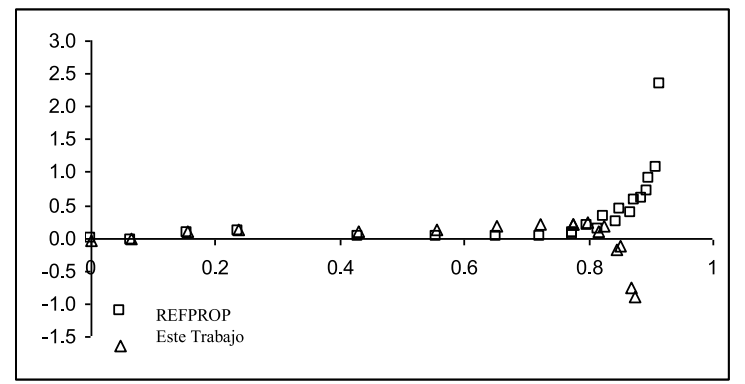

Figura 34. Diferencia para la presión de rocío comparada contra datos experimentales [46] a 449.8K.

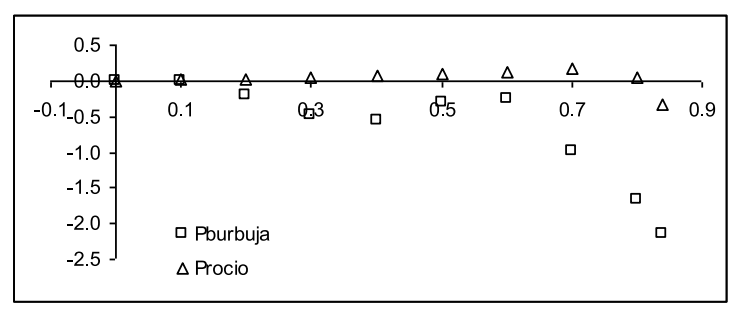

Figura 35. Diferencia de presiones comparada entre modelos a $449.8 \mathrm{~K}$ 


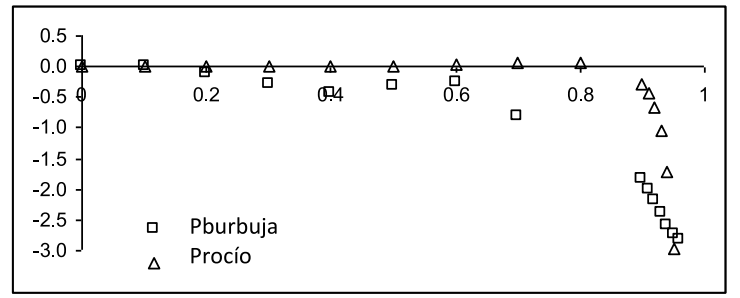

Figura 36. Diferencia de presiones comparada entre modelos a $423.15 \mathrm{~K}$.

\section{REFERENCIAS}

[1] A. Rivera, J. Cerezo, R. Rivero, J. Cervantes y R. Best, "Single Stage and Double Absorption heat transformers used to recover energy in a distillation column of butane and pentane", Int J of Energy Research, vol. 27, pp. 1279-1292, 2003. doi: https:// doi.org/10.1002/er.943

[2] A.I. Kalina, "Combined cycle and waste-heat recovery power systems based on a novel thermodynamic energy cycle utilizing low-temperature heat for power generation", ASME paper n. 83-JPGC-GT-3, 1983.

[3] S. Stecco y U. Desideri, "A thermodynamic analysis of the kalina cycles: comparisons, problems and perspectives". Presentado en Gas Turbine and Aeroengine Congress and Exposition ASME, 1989.

[4] J. Hernández, C. Heard y R. Rivero, "Exergoeconomic comparison of a combined cycle cogeneration system with absorption refrigeration turbine inlet air cooling. Presentado en 16th International Conference on Efficiency, Costs, Optimization, Simulation and Environmental impact of energy systems. Copenhagen, Denmark, ECOS, 2003.

[5] R. Tillner-Roth y G. Friend, "Survey and Assesment of available measurements on thermodynamic properties of the mixture \{Water+Ammonia\}", J Phys Chem Ref Data vol. 27, n. 1, pp. 45-61, 1998. doi: https://doi.org/10.1063/1.556014

[6] E. Thorin, "Thermophysical properties of ammonia-water mixtures for prediction of heat transfer areas in power cycles", Int J Thermophys vol. 22, n.o 1, pp. 201-214, 2001. doi: https://doi.org/10.1023/A:1006745100278

[7] R.A. Macris, B.E. Eakin, R.T. Ellington y J. Huebler, "Physical and thermodynamic properties of ammonia-water mixtures" Research Bulletin Inst. of Gas Technology, n.o 14, 1964.
[8] R.T. Ellington, G. Kinst, R.E. Peck y J.F. Reed, "The absorption cooling process", Research Bulletin Institute of Gas Technology, 1957.

[9] R. Tillner-Roth y G. Friend, "A Helmholtz free energy formulation of the thermodynamic properties of the mixture $\{$ Water+Ammonia\}" J Phys Chem Ref Data, vol. 27, n.o 1, pp. 63-96, 1998. doi: https:// doi.org/10.1063/1.556015

[10] S.H. Risvi y R.A. Heidemann, "Vapor-Liquid equilibria in the ammonia-water system", J Chem Eng Data, vol. 32, 183-191, 1987. doi: https://doi. org/10.1021/je00048a017

[11] A. Vidal, R. Best, R. Rivero y J. Cervantes, "Analysis of a combined power and refrigeration cycle by the exergy method", Energy, vol. 31, pp. 34013414, 2006. doi: https://doi.org/10.1016/j.energy.2006.03.001

[12] J. Pátek y J. Klomfar, "Simple functions for fast calculations of selected thermodynamic properties of the ammonia-water system", Int J Refrig, vol. 18, n.o 4, pp. 228-234, 1995. doi: https://doi.org/10.1016/0140-7007(95)00006-W

[13] K. Sadhukhan, A.K. Chowdhuryi y B.K. Mandal, "Computer Based Thermodynamic Properties of Ammonia-Water Mixture for the Analysis of Power and Refrigeration Cycles", International Journal of Thermodynamics, vol. 15, n.o 3, pp. 133-139, 2012. DOI: https://doi.org/10.5541/ijot.375

[14] E. Thorin, "Comparison of correlations for predicting thermodynamic properties of ammonia-water mixtures", Int J Thermophys, vol. 21, n. 4, pp. 853-870, 2000. doi: https://doi. org/10.1023/A:1006658107014

[15] E. Thorin, C. Dejfors, and G. Svedberg, "Thermodynamic properties of ammonia-water mixtures for power cycles", Int J Thermophys, vol. 19, no. 2, pp. 501-510, 1998. https://doi. org/10.1023/A:1022525813769

[16] G.S. Alamdari, "Simple functions for predicting the thermodynamic properties of ammonia-water mixure", IJE Transactions A: Basics vol. 20 n.o 1, pp. 95-104, 2007.

[17] G.S. Alamdari, "Simple equations for predicting entropy of ammonia-water mixure". IJE Transactions B: Applications, vol. 20, n. 1, 97-106, 2007.

[18] A.A. Zatorskii, "Algorithm for calculation of the parameters of the junction points of the cycles of absorption-type water-ammonia refrigeration machines in a digital computer", Plenum Publishing Corporation, pp. 716-719, 1979. 
[19] K.E. Herold, K. Hain y M.J. Moran, "AMMWAT: A computer program for calculating the thermodynamic properties of ammonia and water mixtures using a Gibbs Free Energy formulation", ASME vol. 4, pp. 65-75, 1988.

[20] Y.M. Park y R.E. Sonntag, "Thermodynamic properties of ammonia-water mixtures: a generalized equation-of-state approach", ASME Trans, vol. 97, pp. 150-159, 1991.

[21] S.N. Mumah, S.S. Adefila y E.A. Arinze, "Properties generation procedures for first and second law analyses of ammonia-water heat pump system", Energy Convers Mgmt, vol. 35, pp. 727736, 1994. doi:https://doi.org/10.1016/01968904(94)90058-2

[22] A. Nowarski y D.G. Friend, "Application of the Extended Corresponding States Method to the Calculation of the Ammonia-Water Mixture Thermodynamic Surface", International Journal of Thermophysics, vol. 19, pp. 1133-1142, 1998. doi: https://doi.org/10.1023/A:1022641709904

[23] R.M. Enick, G.P. Donahey y M. Holsinger, "Modeling the High-Pressure Ammonia-Water System with WATAM and the Peng-Robinson Equation of Sstate for Kalina Cycle Studies", Ind Eng Chem Res, vol. 37, pp. 1644-1650, 1998. doi: https://doi. org/10.1021/ie970638s

[24] L.A. Weber, "Estimating the virial coefficients of the ammonia + water mixture", Fluid Phase Equilibria, vol. 162, pp. 31-49, 1999. doi: https://doi. org/10.1016/S0378-3812(99)00181-8

[25] F. Xu, y D.Y. Goswami, "Thermodynamic properties of ammonia-water mixtures for power-cycle applications", Energy, vol. 24, pp. 525-536, 1999. doi: https://doi.org/10.1016/S0360-5442(99)00007-9

[26] R. Sharma, D. Singhal, R. Ghosh y A. Dwivedi, "Potential applications of artificial neural networks to thermodynamics: vapor-Liquid equilibrium predictions", Computers and Chemical Engineering, vol. 23, pp. 385-390, 1999.doi:https://doi. org/10.1016/S0098-1354(98)00281-6

[27] R. Lugo, J. Guilpart y L. Fournaison, “Calculation method of thermophysical properties of ammonia-water mixtures", Presentado en Second Workshop on Ice Slurries, Paris France, International Institute of Refrigeration, 2000.

[28] A.A. Vasserman, A.G. Slynko, S.V. Bodyul, Yu.V. Gondarenko y E.S. Bodyul, "A Thermophysical Property Databank for Technically Important Gases and Liquids", International Journal of Thermo- dynamics, vol. 22, pp. 477-485, 2001. doi: https:// doi.org/10.1023/A:1010774831521

[29] R. Lugo, L. Fournaison, J.M. Chourot y J. Guilpart, "An excess function method to model the thermophysical properties of one-phase secondary refrigerants", International Journal of Refrigeration, vol. 25, pp. 916-923, 2002. doi: https://doi. org/10.1016/S0140-7007(01)00105-0

[30] R. Span y W. Wagner, "Equations of State for Technical Applications. I. Simultaneously Optimized Functional Forms for Nonpolar and Polar Fluids", Int J of Thermophysics, vol. 24, pp. 1-39, 2003. doi: https://doi.org/10.1023/A:1022390430888

[31] R. Span y W. Wagner, "Equations of State for Technical Applications. III. Results for Polar Fluids", Int J of Thermophysics, vol. 24, pp. 111-162, 2003, doi: https://doi.org/10.1023/A:1022362231796

[32] M. Barhoumi, A. Snoussi, E.N. Ben, K. Mejbri y A. Bellagi, "Modélistion des données thermodynamiques du mélange ammoniac/eau", Int J Refrig, vol. 27, pp. 271-283, 2004. doi: https://doi. org/10.1016/j.ijrefrig.2003.09.005

[33] Kh. Mejbri y A. Bellagi, "Modelling of the thermodynamic properties of the water-ammonia mixture by three different approaches". Int J Refrig, vol. 29, pp. 211-218, 2006. doi: https://doi.org/10.1016/j. ijrefrig.2005.06.002

[34] A. Sencan, "Artificial intelligent methods for thermodynamic evaluation of ammonia-water refrigeration system", Energy Conv \& Man, vol. 47, pp. 3319-3332, 2006. doi: https://doi.org/10.1016/j. enconman.2006.01.002

[35] A.H. Farrokh-Niae, H. Moddarress y M. Mohsen$\mathrm{Nia}$, "A three-parameter cubic equation of state for prediction of thermodynamic properties of fluids". J Chem Thermodynamics, vol. 40, pp. 84-95, 2008. doi: https://doi.org/10.1016/j.jct.2007.05.012

[36] N.S. Ganesh y T. Srinivas, "Evaluation of thermodynamic properties of ammonia-water mixture up to 100 bar for power application systems", Journal of Mechanical Engineering Research, vol. 3, no. 1, pp. 25-39, 2011.

[37] S. Kherris, M. Makhlouf, D. Zebbar y O. Sebbane, "Contribution study of the thermodynamics properties of the ammonia-water mixtures", Thermal Science, vol. 17, n.o 3, pp. 891-902, 2013. doi: https://doi.org/10.2298/TSCI110206083K

[38] F. Li, L. Duanmu, L. Fu y X.L. Zhao, "Research and application of flue gas waste heat recovery in cogeneration based on absorption heat-exchange", Proce- 
dia Engineering, vol. 146, pp. 594-603, 2016. doi: https://doi.org/10.1016/j.proeng.2016.06.407

[39] A. Modi y F. Haglind, "A review of recent research on the use of zeotropic mixtures in power generation systems", Energy Conversión and Management, vol. 138, pp. 603-626, 2017. doi: 10.1016/j. enconman.2017.02032

[40] A. Rattner y S. Garimella, "Fast, stable computation of thermodynamic properties of ammonia-water mixtures", International Journal of Refrigeration, 2015. doi: https://doi.org/10.1016/j. ijrefrig.2015.09.009

[41] M. Wang, A. Manera, S. Qiu y G.H. Su, "Ammonia-water mixture property code (AWProC) development, verification and Kalina cycle design for nuclear power plant", Progress in Nuclear Energy vol. 91, pp. 26-37, 2016. DOI: 10.1016/j.pnucene.2016.04002

[42] O.M. Ibrahim y S.A. Klein, "Thermodynamic properties of ammonia-water mixtures", ASHRAE Trans, pp. 1495-1502, 1993.

[43] Y.M. El-Sayed y M. Tribus, "Thermodynamic properties of water ammonia mixtures theoretical implementation for use in power cycles analysis", Special publication AES New York, ASME, n. 1, pp. 89-95, 1985.

[44] B. Ziegler y Ch. Trepp, "Equation of state for ammonia-water mixtures", Int J Refrig, vol. 7, pp. 101-106, 1984. doi: https://doi.org/10.1016/01407007(84)90022-7

[45] S.C Chapra y R.P. Canale, "Métodos numéricos para ingenieros". Quinta Edición (Español), Mc Grau Hill Interamericana, México, 2007.

[46] P.C. Gillespie, W.V. Wilding y G.M. Wilson, "Vapor-Liquid equilibrium measurements on the ammonia-water system from $313 \mathrm{~K}$ to $589 \mathrm{~K}$ ". AlChE Symp Ser, vol. 83, n. 256, pp. 97-127, 1987.

[47] J. Pospisil y Z. Fortelny, "Graphical expression of thermodynamic characteristics of absorption process in ammonia-water system", EPJ Web of Conference, vol. 25, 01079(1)-01079(8), 2012. DOI:10.1051/eojconf/20122501079.

[48] A. Sencan, S. Gök y E. Dikmen, "Prediction of liquid and Vapor Enthalpies of Ammonia-water Mixture",
Energy Source, Part A, vol. 33, pp. 1463-1473, 2011. doi: https://doi.org/10.1080/15567030903397891

[49] K.H. Kim, K. Kim y H.J. Ko, "Entropy and exergy analysis of a heat recovery vapor generator for ammonia-water mixtures", Entropy, vol. 16, pp. 20562070, 2014. DOI: 10.3390/e16042056

[50] R.C.E. Campos, P.J.C. Escobar, S.C. Rodríguez, M. Leme, O.J. Venturini, L.E. Silva, C.V. Melián, D. dos S. Marques, D.F.R. Lofrano y V. Gialluca, "Exergetic and economic analysis of Kalina cycle foor low temperatura geotermal source in Brazil", Presentado en Procedings of ECOS 2012. The 25th International conference on efficiency, cost, optimization, simulation and environmental impact of energy systems. Perugia, Italy, 2012.

[51] V. Srikanth, B.R. Narender y A. Gupta, "Thermodynamic Analysis of vapour absorption refrigeration system using solar energy", International Journal of Latest Trends in Engineering and Thechnology, vol. 7, n.o 4, pp. 17-26, noviembre 2016. DOI: 10.21172/1.74003

[52] L. Becker y C.J.L. Corrales, "Fundamental EoS Implementation for $\{$ Water+ammonia\} in Modelica", Presentado en Proceedings of the 11th International Modelica Conference, September 21-23, pp. 647-652, 2015. doi: https://doi.org/10.3384/ ecp15118647

[53] M.A.I. El-Shaarawi, S.A.M. Said y M.U. Siddiqui, "New Correlation Equations for Ammonia-Water Vapor-Liquid Equilibrium (VLE) Thermodynamic Properties", ASHRAE, DA-13-025, 2013.

[54] D. Kong, J. Liu, L. Zhang, H. He y Z. Fang, "Thermodynamic and Experimental Analysis of an Ammonia-Water Absorption Chiller", Energy and Power Engineering, vol. 2, pp. 298-305, 2010. doi: https:// doi.org/10.4236/epe.2010.24042.

[55] L. Luo, H. Gao, Ch. Liu y X. Xu, "Parametric investigation and Thermoeconomic Optimization of a Combined Cycle for Recovering the Waste Heat From Nuclear Closed Brayton Cycle", Science and Technology of Nuclear Installations, 2016. doi: https://doi.org/10.1155/2016/6790576

[56] R. Rivero, G. Montero y R. Pulido, "Terminología para la Aplicación del Método de Exergia", Revista del IMIQ, vol. 17, pp. 7-11, 1990. 